\title{
A REVIEW OF MODERN ELECTRICAL THEORIES.
}

BY PROFESSOR WM. A. ANTHONY.

The general phenomena of electricity are familiar to us all. As children we learned of attraction and repulsion of electrostatic charges, of condensers, of insulators and conductors, of electric currents and electro-magnets, of telegraphs, and electric fire alarms and electric bells, of the heating of conductors, and even electric lights. We have long been familiar with the effect of the current on a magnetic needle, and with the attraction and repulsion of currents for each other. We have seen that these effects are manifested at a distance from the conductors which carry the currents, and we have been willing to believe that the distances at which these phenomena could be recognized were limited only by the want of sensitiveness of the apparatus through which the effects were manifested. Probably not many of us stop to think how these effects were brought about. We were content with a general statement of the relations of the phenomena, or, at least, with the determination of the direction and intensity of the forces due to a given conformation of current. We talked of the attractions and repulsions as action across space, and, if we thought of them at all as requiring any mechanism through which to act, we still felt satisfied when we had discovered and formulated the laws that govern the action of these forces.

It did not matter to us that attraction across empty space was unthinkable; it was enough that action took place, and that we could measure and, to a certain extent, control it. We constructed instruments and computed forces acting under certain conditions; we multiplied experiments and thought we had developed a vast science. But there have not been wanting, even among the earlier philosophers, some who could not feel satisfied with 
the idea of action at a distance. Experience gives no clue to any explanation of action of one body upon another at a distance, except through some intervening mechanism, and if we find cases where such intervening mechanism is not apparent, to say that in these cases action at a distance is taking place, is only to say that the action is unexplained.

Gravity acts upon the distant planets, and, we believe, upon the most distant stars; but it is not enough to say that gravity acts, that it decreases as the square of the distance increases, we want to know how it acts. By what means or through what medium does our earth reach out to the distant planets and affect their movements? These are the questions that the dissatisfied few have asked in the past, and to-day it is not the few but the many who would be glad to have the question answered. Faraday early taught us that the mere statement of the law of electrical attraction or repulsion was no explanation, and he sought the explanation in the action in the intervening medium. He demonstrated by experiments, that have become classic, that the effect of induction depends upon the nature of the medium intervening between the conductors; a demonstration that here, at least, the intervening medium took part in the action and helped to determine the result. Magnetic and electro-magnetic phenomena were to Faraday also effects propagated through the medium; and Maxwell, following Faraday's work, subjected the whole matter to a searching mathematical analysis. It was necessary, in order to complete the theory, to assume the existence of a medium in which the forces were exerted; but, as a medium had already been assumed to account for the phenomena of light, it would hardly be scientific to assume another to account for electrical phenomena. But would not one medium suffice for electrical and optical phenomena as well? Maxwell showed how the question of the identity of the two media could be tested, and our modern views of electricity are mainly the results of his work. What do we know of electrical phenomena? We know that pieces of glass and silk rubbed together and then separated attract each other ; that the property of attraction can be imparted to metals if they are insulated; that if two such masses of metal be connected by a wire the power of attracting each other disappears, and at the same time, the wire is warmed and for an instant exerts magnetic forces at right angles with the wire. Numerous other facts we know, but these will suffice as illustrations of the 
phenomena. When a metal conductor is discharged by touching it with a wire we say the wire carries a current, and we have been accustomed to ascribe the heating and the magnetic effects to that current. We have looked upon the thing, whatever it is, that takes place in the wire as the origin of all that takes place around it. The current warms the wire, and it confers upon it the property of acting upon magnetic needles; but how does it act? We cannot conceive of the action of that wire on that needle, except through something extending from the wire to the needle through which the wire can exert a push or pull. All around that wire the needle is affected; at considerable distances the effect can be exhibited. and we believe it is only want of delicacy of our apparatus that prevents our discovering it at the greatest distances. If the wire acts it acts through a medium in which it

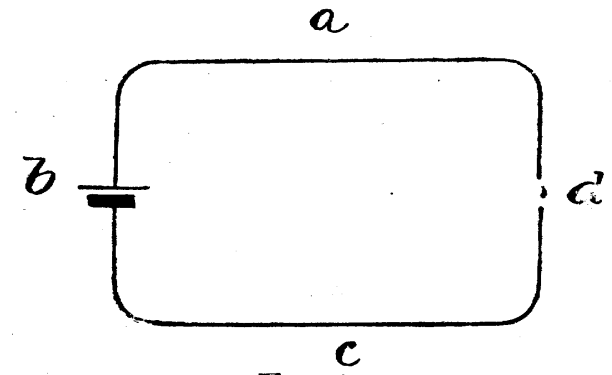

FIG. 1.

creates a disturbance, and the disturbance in turn acts upon the magnetic needle. But are we sure that the action begins with the wire? Is it not possible that the action begins in the medium, and that the wire merely separates portions of the medium and so permits action that would not otherwise take place? Maxwell has proposed a mechanical illustration of the action taking place in space when electrical phenomena occur, and Fleming and Lodge have elaborated it and brought it within the reach of nonmathematical readers. Let us suppose all space to be filled with wheels connected by idle wheels in such a way that when one turns all must turn in the same direction, except that the elasticity of the system permits a small movement of one wheel before the others in the vicinity begin to move. Electromotive force is then something that tends to turn the wheels. Let $b$ in Fig. 1, represent some source of electromotive force; $a$ and $c$ conductors not forming a closed circuit but having an opening at $d$. The electromotive force at $b$ tends to turn the wheels, but so 
long as the gap exists at $d$ the wheels are connected through all space, and the E. M. F. at $b$ can effect nothing except to turn the wheels through a small angle until the elastic resistance balances the E. M. F. In the conductor, the wheels are not supposed to be rigidly connected. They can turn by slipping on each other, and the wheels in space may turn by slipping on the wheels in the conductor; hence, as the ends of $a$ and $c$ approach each other, the connection between the wheels within the enclosure and those of outside space becomes smaller and smaller, and, when the conductors touch, there is no longer anything to prevent the wheels within from turning, and they begin to spin in obedience to the E. M. F. at $b$. The conductor is heated by the friction due to the slip of the wheels around and within it. The spinning of the wheels constitutes the magnetic forces if we assume the wheels, originally spherical, to become flattened spheroids by the spinning, and to be forced to remain in contact at their poles. Assume the wheels to have inertia, and it will require time for them to reach their full velocity, and the time will be greater as the number of wheels is greater, that is, as the magnetic forces developed are greater. On the other hand, the wheels will not at once stop if the electromotive force is removed, and if the conductor be broken at some point, allowing the rapidly spinning wheels enclosed by it to come in gear at that point with the stationary outside wheels, there will be, for a brief time, until the moving wheels can be brought to rest, a clashing and disruptive action known as the extra spark.

I have spoken of the heating of the conductor as due simply to the friction of the spinning wheels upon the wheels within the conductor. The greater the friction the greater the motion imparted to the wheels within the conductors and the greater the heating. The less the friction the less the heat, and if there were a substance whose wheels could revolve among themselves and against the wheels of space without friction, such substance would form a perfect conductor which would offer no resistance to the motion of the wheels in the space inclosed by it, and however rapidly those wheels might revolve they would have no influence to produce motion within such a conductor. We are led, then, to this, that a perfect conductor, instead of being a substance in which any E. M. F. produces an infinite flow of electricity, may be a substance in which no effect whatever is produced.

I do not present this as representing the real activities involved 
in electrical phenomena, or as a theory by which all electrical phenomena may be explained. I give it as bringing prominently into view the activities in the medium surrounding the conductor; whereas, the language in which we usually describe electrical phenomena points to the conductor itself as the seat of the electrical forces. Whatever our theory, we know that something does take place in the medium that surrounds the conductor. We know that were a heavy current to flow through a conductor in the most distant part of this room a delicate needle here on the table would show that a magnetic field was produced here. We are certain that magnetic lines of force from the electric currents on our streets, whether overhead or underground, permeate this room, and traverse our bodies even, though we have no sense through which we can become directly conscious of them. Take in your hands two wires connected to the poles of a battery or dynamo and touch their ends together. You see a flash of light, the wires become warm, they attract iron filings; coiled into a spiral they will attract with great force large masses of iron; two such spirals will attract or repel one another. What more natural than to say: Here in the wire is a new activity which warms it and confers upon it magnetic properties? What more natural than to forget or fail altogether to recognize the activities in the space around; activities that do not impress any of our senses; that do not wrench our arms, or burn our flesh, or blind our eyes; and yet it is well to fix our attention for a while upon the space that the conductor encloses, and recognize the fact which the little delicate needle demonstrates to us, that something is going on in that space. something quite as important, perhaps more important, than that which takes place in the conductor.

Whatever the magnetic forces are, we know they cannot be called into existence instantaneously. Time must always elapse before the full effect of an electric current is felt, and the greater the forces developed the longer are they in reaching the maximum. Moreover, the current in the wire cannot be at once developed; it keeps pace with the magnetic force and reaches a maximum only when the magnetic forces reach a maximum. This is exactly what would follow if magnetic forces result from the setting in motion of wheels possessing inertia. Whatever magnetic forces may be, they begin and end like the motions of massive bodies. 
Continuing our hypothesis, an electromotive force would be anything that tends to set the wheels in motion. The wheels are set in motion if a limited number acted upon by the E. M. F. are separated by a conductor from the other wheels of space; but, even if they are not separated, that is, if the conductor does not. form a closed circuit, the wheels are rotated through a small angle depending on the elasticity of the wheelwork. This displacement constitutes an electric charge, the tendency of the wheelwork to spring back is difference of potential, and this difference of potential is maintained by the E. M. F. Here allow me to call attention to the distinction between difference of potential and E. M. F. I often see statements that indicate that the distinction is not clearly kept in view. In that excellent little volume, the Dictionary of Electrical Terms, the statement occurs that electromotive force is due to difference of potential. It should

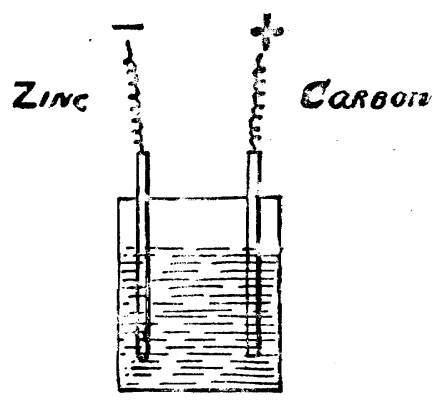

Fig. 2.

rather be difference of potential is due to E. M. F. Difference of potential is an electromotive force, but not all electromotive forces are difference of potential; and, furthermore, I can think of no case of difference of potential that does not owe its existence to some other electromotive force, not difference of potential. Difference of potential is a moving force acting from within. Electromotive force acts from without. Difference of potential results from a changed electrical distribution, an electric strain, and represents the tendency to return to the state of equilibrium. Electromotive force is the something from without that produced the electric strain. Consider our wheelwork illustration. I may seize a wheel here and turn it, but I can only turn it through a small angle before the elastic forces, brought into. play by the distortion, balance the force that I am able to exert. My force is electromotive force, the elastic forces are difference 
of potential. Let Fig. 2 represent a voltaic cell with its copper and zinc plates and dilute acid; we know that something takes place by which the copper becomes positive and the zinc negative, and, if the copper and zinc be joined by a wire, electricity is said to flow from the copper to the zinc through the wire, and from zine to copper through the liquid. The flow through the liquid used to be explained by assuming the zinc to be positive and the copper negative within the liquid; just the reverse of what we find without. But connect the copper to the zinc by a wire under the liquid, the current flows through the wire from the copper to the zinc. The copper, therefore, is not negative, but everywhere positive. Then why does the current set from the zinc to the copper through the liquid? Becanse there is an electromotive force, not difference of potential, mind you, for the difference of potential tends to cause a flow the other way, but a something that forces the current through the liquid against the electric pressure. The cell is an electric pump that pumps electricity from the zine into the copper until the pressure becomes so great that the pump stops. Open a passage from the copper to the zinc by connecting the two by means of a metallic wire, the electricity flows and relieves the pressure, the pump begins to work and so maintains a continuous flow. No; E. M. F. is not difference of potential. although difference of potential is E. M. F. It is even possible to produce an electric current without any difference of potential whatever in any part of the circuit, just as it is possible to produce a flow of fluid without any difference of pressure.

Whatever difference of potential may be, it behaves like a force developed by an elastic displacement in a solid body. Whatever magnetic forces may be, they behave as though produced by the motions of inert masses. An electromotive force, suddenly brought to bear at a given point, has to overcome not only the elastic force but the magnetic inertia. In other words, an electromotive force, tending to produce current, immediately develops a difference of potential against which it must act, and also develops magnetic forces which retard its action; so a fluidmoving force meets opposition in the elastic force of the fluid and in the inertia of its particles. But the effect of any such force is propagated through the medium with a finite velocity expressed by the well-known formula. 
$$
V=\sqrt{\frac{E^{\prime}}{D}}
$$

Electric displacements ought, therefore, to be propagated with a finite velocity which should have some relation to the ratio between the electrostatic effects, analogous to elastic displacements, and the electro-magnetic effects analogous to the effects of moving masses. You all know that it is shown that this velocity is expressed by the ratio of the magnitudes of the electro-magnetic and electrostatic units quantity, and you know also that determinations of this ratio show it to be the velocity of light. This determination gave a very strong support to Maxwell's electro-magnetic theory of light, and, coupled with the fact that observations, so far as they went, indicated that magnetic effects are propagated from the sun to the earth with the same velocity as light, made the theory at least extremely plausible. But within two years the wonderful experiments of Hertz have demonstrated beyond question that electro-magnetic waves travel through space from every source of alternating currents or potentials, and that the waves travel with the velocity of light. In this city there are, I suppose, many alternating current systems. When we think of these in action we are apt to think only of the activity in the conductors of the machines, the lines, the transformers, the lamps, and yet we know that in all the space around there is activity. Waves are chasing each other through this room, through our streets, our houses, our offices. They are everywhere present. We are bathed in this agitated medium every moment, and yet we live, and not only live, but are totally unconscious of the activity that surrounds us. No sense responds to the wave motion that fills this space. And yet, when these waves become short enough and frequent enough, they do affect our sense of vision and a vast array of phenomena that otherwise would have had no existence for us, are made known to us through this special sense. But the eye only responds

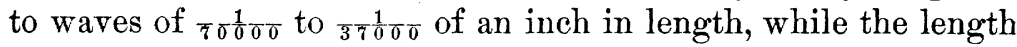
of the waves emitted by one of our alternating current systems is 600 miles. The waves that affect the eye must occur at the inconceivable rate of at least 400 millions of millions per second, while less than 300 waves per second are emitted from our alternating systems. These waves come very far short of the number necessary to affect any of our senses; but we have been able to demonstrate their existence, at least we have been able to show 
that something possessing all the characteristics of wave motion exists in the space around a source of alternating currents or potentials. It is something to know that waves of such enormously different lengths and frequencies exist in the medium that is agitated by waves of light. It is something to understand that our sources of alternating currents are centres of radiant energy, differing from light only in wave length; and since we have begun to appreciate this fact, we have often asked ourselves the question: Can the rate of alternation be increased until the whole apparatus should glow with light? Although the enormous rapidity required seems to render this direct solution impossible, it seems to me there must be a way to obtain the light we want without all this waste of energy. I cannot believe it will always be necessary to develop waves of all lengths from many miles down to a hundred thousandth of an inch, in order to obtain the narrow range of wave lengths by which we see. I do not know of any practical way of obtaining the few wave lengths that constitute light without at the same time producing the others, but it is done. The glow-worms do it, the fire-flies do it, the lantern beetles do it, and I believe the time is coming when man can do it. Instead of getting 10 16-candle lamps per horse-power we ought to get 200 . I don't know how it is to be done. I don't expect we are going to make alternating machines to produce 500 millions of millions of alternations per second. But possibly we may solve the problem indirectly by the use of some substance having a special rate of vibration, such as the gases. Possibly we may be able to exite electrically the fluorescent salts. Possibly we may be able to charge and discharge a condenser and take advantage of the oscillatory discharge to set up vibrations of the frequency required. Possibly we may discover the secret of the glow-worm and fire-fly and substitute electric for the insect energy. I know it will take several fire-flies to equal a 16-candle lamp, but it will also take a good many fire-flies to develop a horse-power. But although I do not see at present any practical solution of the problem, I repeat, I believe the problem can and will be solved. We are not going on forever burning a coal mine whenever we want a little light. Neither are we going on forever converting the energy of coal into heat when it is mechanical or electrical energy we want. From the very nature of things not more than one-fourth or one-third the heat so produced can be transformed into any other form of energy. The energy of 
fuel can be converted into mechanical energy without first becoming heat. It is so converted in every animal movement. The way exists; let us find it. Find this, and the way to make light only, when it is only light we want, and we shall have lengthened the life of our coal deposits five or ten times. The man who solves either one of these problems will be the greatest discoverer of this or any other age. If he gets a broad patent on it, he will have seventeen years of the grandest litigation the world ever saw.

Now I can imagine that some electrician will consider that the first part of this paper dealt with matters that are of no practical consequence in the use of electric currents. Of what practical consequence is it whether force is conveyed to a distance by means of a medium or simply acts at a distance. What does it matter whether electric energy is conveyed by the conductor or through the dielectric? We know how to proportion and dispose our conductors to produce a given effect, and that is enough. But consider what we have learned already of the intimate connection between light and electricity, and I think you will agree that the more we know of the ultimate nature of electricity the nearer we shall be to the solution of the great problem to which I have referred, and the solution of either one of those problems would revolutionize some of the most important industries of the world. May not the solution of at least one of these problems be accomplished and the results exhibited at the great exhibition to be held in 1892? What a wonderful achievement it would be to light all those great buildings without heat, or drive all that vast array of machinery without a fire!

\section{DISCUSSION.}

The Chairman, (George M. Phelps):-Professor Anthony has very pleasanty led us into a realm of speculation--not altogether speculation, because it is founded on many things known before, and I am quite sure that some of you will be glad to follow him immediately in treating of the topic that he has so happily opened.

Dr. Otтo A. Moses:-I have to thank Professor Anthony for a most interesting lecture. It has indeed set our thoughts moving. I presume that all of us here have been very deeply interested in those speculations, because though they may be speculations, they are the scaffolding upon which the whole science of electricity has been based. It was the pursuit of a hypothesis that led 
Kepler year after year through very patient investigations to the results which revolutionized astronomy. The same course led Faraday, step by step, to those determinations which finally culminated in what we are now first recognizing - the identity of electricity and light. So that I can say that we have all been gradually accustoming ourselves to these speculations, even though many of us may be engaged in the practical uses of electricity.

There are some remarks in Professor Anthony's very interesting observations to which I would like to call particular attention, more especially the last, as bearing upon our practical applications of these speculations. He spoke of the possibility of using the molecular action of gases as a means of transforming wavelengths. If my memory serves, that has been done ever since the Ruhmkorff coil has been in cperation and the Geissler tube has become so perfect an analyzer of the electric current. In that respect we have already anticipated some of these results. Let us consider what we already have. In the Geissler tube you have the attenuated gases; you have two disconnected poles; you have a current passing, and the wave-lengths broken up and the light produced. So that we have right there before us the facts. Whether we can ever utilize the Geissler tube to effect these immense economies that the Professor so pleasantly prophesied is a question to be determined. It has been experimented upon, and $I$ have found in my short experience in life that these things come when they are needed. There are certain groups of ideas that must be exhausted before people will adopt others and investigate them. We have seen it in the development of all the different branches of electric appliances. We have seen it in the telegraph. We have seen, for instance, Morse using Wheatstone's method of transmission; we have seen him abandon it. We saw him commence with underground conductors; we saw him abandon them simply because the art of that day was not perfect enough to allow him to supply them with profit; so that we can unearth many and many a theory now which already existed, but only awaited the development of the arts to be put into practice.

Now there is one thing I would like to add as a brief supplement to Professor Anthony's predictions. It is in no critical spirit that I mention it, because I will be running somewhat counter to the mechanical hypothesis upon which he developed many of his remarks. One, with great humility, must undertake to oppose himself to a theory emanating from such a mind as: that of Clerk Maxwell. but one of the difficulties in the minds of all great mathematicians is that they must put their thoughts into mechanical methods in order to be able to weigh and measure directly. If we could only divest ourselves of the fact that they considered all nature to be composed of molecules, we would then come into another range of thought. I see myself no necessity for imagining all nature split up into infinite particles- 
infinite in number and infinitely small and large in size. I can readily conceive of a continuity of matter. In fact, that thought is no novelty. It has been woven through all science, only we are so accustomed to limitations in our contact with nature, that we are apt to conceive that those limitations actually exist beyond our mere perception of them. As Professor Anthony said, very properly, there is an immense difference in our sense of observation between a wave length of electricity and nne of light, but there is no reason that there is not a perfectly insensible graduation from the wave of electricity into that of light. In fact we see it when the current changes from heating effect into light.

A Member:- There is one point I would like to have explained in the wheel hypothesis: To increase the current of a conductor without increasing the heat waste, it is necessary to increase the area of the conductor. I should think that would increase the number of wheels inclosed in a conductor, and therefore increase the friction and the amount of slip, and thus increase the heat developed instead of decreasing it.

Professor Anthony:-As I understand it, the more wheels there are in a conductor, the less is the amount of energy spent in causing the slip over them, because you have got more surface for slip. What we call a given current is accompanied by certain magnetic effects That would mean that the wheels in space enclosed by the conductor are revolving at a certain rate. Now supposing those all to be revolving at the same rate and increasing the size of the conductor and increasing the number of wheels there are in it, the wheels that are in the conductor will be set revolving at a less rate instead of a greater one, because there will be greater surfaces where the slip can occur.

P.ofessor M. M. Garver:- - It is rather curious to me that generally speaking all the heat developed in the conductor, as Mr. Heaviside looks at it is an action around the conductor. In that connection I cannot see, if it takes place around the conductor, why the law of temperature should hold as it does in the case of metals. Take for instance, the case of copper; I believe the variation is about two tenths of , ne per cent. for one degree Fahrenheit. If the same law holds, for $500 \mathrm{deg}$. below zero Fahrenheit there should be no resistance and no heat developed in the conductor. I believe some experiments have been made in regard to the conductivity of copper at very low temperatures. I would be glad to hear from any one who has knowledge of this subject.

Mr. A. F. Kenneldy :-I think it would be unnecessary for me to add my own personal views to what I am sure is the universal sentiment concerning the paper to which we have had the pleasure of listening. But while it is full of food for reflection from both a theoretical and practical point of view, I hope there is one point more that may be permitted to be brought forward, that is, the great difference between what might be called a working theory and an ultimate theory. Tracing back the history of any of the 
sciences, as they are so called to-day, we see how very wide-spread they were, how numerous the sciences were, how in the Greek days every activity such as the motion of a cloud and the motion of a body in the air, was supposed to proceed from some superior cause, and gradually as knowledge increased and as investigation pursued its quiet path, those forces were singled out and found to be fewer and fewer, and so to-day, we stand with a certain number which we still hope will be reduced and finally embodied in one. Dr. Moses has given us a very beautiful, I might almost say, a poetic view of what he calls the infinitude of nature, but I think it would be a finer expression perhaps, if he will allow me the correction, if that infinitude be considered as proceeding rather from the unity of nature. In fact if we go back to the days of Polytheism, the old Mahometan cry "There is one God" stands very aptly by comparison. In the theory of heat for example there were what we call now the crudest notions prevalent about four hundred years ago as to what its nature might be. Slowly those views have become clearer, and now perhaps heat is in its theory more complete than any of our other sciences. But the trouble with heat has been the same as it is now with electricity-the difficulty of getting first a theory that was workable and then a theory which would account for the facts clearly and consistently. We have listened to the explanation of a beautiful theory, a theory worthy certainly of the master minds that originated it; but, somehow it does not appear as though that theory were going to be the ultimate one which would satisfy us when we come to consider nature as a whole, and that although it is a good working hypothesis-a peg upon which we can hang our thoughts- that after all we may hope for some simpler and more universal distribution of matter than spinning wheels in space. A vacuum was once supposed to be the absence of everything from space, and nature, as we read in the old books abhorred a vacuum but gradually people began to find out there must be something left, because phenomena take place which could not be accounted for if there were nothing there, for we cannot conceive of action through nothing, and gradually as light came to be considered no longer on a corpuscular theory, it became necessary to invent a new ether and finally the question arose, will not the same ether do for both? Then came the electro-magnetic theory of light and that required a special ether, but that also has been united. Now for the brilliant work of Hertz and Maxwell we see that those two ethers are one and the same; at least we cannot distinguish between their properties. The ether that we suppose will propagate light, is surely not one that we can readily unite with the idea of spinning wheels in space. The mere fact that the thing is difficult to conceive of, is no bar of course to its truth. But we are entitled to the expectation that nature in her infinity is infinitely simple. Surely, it is in this ether that we have to in- 
vestigate something which unites more readily the functions of gravitation of light and of electricity. If we have, for example, a vessel full of incompressible fluid-water will answer-and we introduce two little jets, small by reference to the whole vessel, and we cause the vessel to be hermetically sealed, full of water, then if we force water out of one jet, back through the other, causing a circulation to take place, we shall find, if there be no vortex-motion in the liquid, and if all the particles advance in ranks, shoulder to shoulder-that the lines pursued by the individual particles are, strangely enough, the lines which are followed, we have every reason to believe, by magnetism, by heat and by gravitation. Surely there is some grand principle under lying that great class of phenomena which some day in the future will join them all into one coherent principle.

The point that Prof. Garver mentioned about the conductivity of copper at a very low temperature certainly has great practical interest although we are not likely to live in a climate where for the sake of our conductors we submit to two or three hundred degrees below zero But I think that experiments have been made at minus 40 degrees centigrade which showed a very large increase in the conductivity of copper, and the co-efficient of temperature was very much larger at that point than it was higher up the scale. The late Sir William Siemens had a very interesting theory that the resistance at a very low temperature was very small indeed, but rose rapidly at first and slower and slower afterward. That is contrary to our orthodox views about conductivity of copper at high temperatures, but it is a view which is interesting in itself.

Mr. Townsend Wolcotr:-Regarding the existence of a medium, Professor Anthony has given voice to the most general opinion in saying that action at a distance is unthinkable. I believe this is the view which Sir Isaac Newton took; but it must not be forgotten on the other hand that Roger Cotes said that there is no a priori reason for disbelieving in action at a distance any more than any other experimental facts that we have, and it is this same Roger Cotes of whom Sir Isaac Newton said in speaking of his untimely death: "Had he lived we should have known something." I think that a part of the difference between these two classes of thinkers arises from their having a different conception of the meaning of the word medium. In the present state of the science, if we do not make the word medium mean too much, it is in my opinion practically demonstrated that there is a medium. If we say that action at a distance through spacevacant space-is unthinkable, what do we mean by space? If we mean simply extension, the concept of extension being simply three dimensions, it is unthinkable. But in conceiving of a medium we are too apt to make the word mean too much. Now, Professor Stokes has shown that the luminiferous ether acts like an elastic solid; that is to say the mathematics of solid elasticity 
apply to the ether; but in order that it should be an elastic solid which had the same degree of elasticity as the ether does have, on that hypothesis it would have to be an exceedingly solid kind of a solid. I forget how much pressure there would have to be, but it was something enormous. Now, we know that the ether is not such a solid as that. Again taking the modern theory that the action of the dielectric is a stress, we may apply the same comment to this also. The mathematics of stress are equations similar to the equations that apply to electric phenomena in a dielectric." However, there can be no real stress in the sense in which we understand the word, because the very bodies which can not sustain a stress are the ones which are the best dielectrics. In fact a vacuum, which we do not consider a body at all, is a dielectric, and, according to some electricians, it is the very best of insulators; and metals which generally are capable of sustaining a great deal of stress, are all conductors and, according to theory, are bodies in which the state of dielectric stress cannot exist, but gives way immediately, producing what we know as current.

Dr. Michael Pupin:-I would like to express my feeling of obligation to Prof. Anthony for his able lecture on the modern theories, and with his kind permission to add a few comments as a supplement to his review. We know what ideas Faraday had of dielectrics and the function which they perform in the transmission of the electric action from one part of space to another. We know that Faraday supposed the dielectric to be filled with small conducting particles which have the property of polarity. In what that polarity may consist we do not know. We may suppose it is of the same kind as the magnetic polarity in the case of soft iron. That theory has been worked out by Professor Helmholz, and he deduced the equations for the distribution of the stress in the medium of the dielectric exactly the same as Maxwell did. In fact the equations which Helmholz obtained are a little bit fuller. Now, given that a dielectric consists of very small particles, each one having the properties of polarity and all the electro-static and all the electro-magnetic phenomena can be explained. I do not see that it is necessary to go into the consideration of wheels working within other wheels. To be sure Maxwell did work out the theory in 1862, I think, to which reference was made this evening, but he gave it up, and in his second volume on electricity he refers to this theory in a sort of off-hand way, saying that some years ago he worked out a theory of electricity based on the hypothesis of Sir William Thomson of vortex motion. Helmholz, supposing the medium to have only this property of polarization - these papers are not published yet, but I expect they will be published very soon, though they are not very new, because these things have been worked out before by Sir William Thomson, and I think by Maxwell-shows that if there is any electric disturbance, whether it be an electrostatic or electro-magnetic, in the conductor must be propagated 
in the whole dielectric, because disturbance in the electric state of the conductor will cause a disturbance of the stress in the dielectric, and that disturbance of the metal gives the electromagnetic action.

Every one of the speakers this evening has spoken of forces. The term force is very elastic and may mean almost anything. In fact, I think every man has his own idea about force. But if we are to discuss and use the term we must have the same definition. I think if we are to treat sciences from the mechanical standpoint, then we must define force mechanically. That is, we must fall back necessarily on the Newtonian definition of force, $i$. e. as producing nothing but motion-this is the elementary definition of force. When we speak of electric and magnetic forces we certainly deal with something which will produce more than motion alone. Take, for example, Ampere's integral law, by which we know that a current flowing through a conductor will act upon a magnetic pole just the same as if the wire was an empty shell having a magnetic moment equal to the strength of the current. But, in every department of science it is always desirable, and in fact it is the highest aim of every physicist to deduce, not the integral law but the differential law. Newton achieved his great success when he found the differential lawthe law of universal gravitation. Ampere thought his integral law would be of little use to him if he did not know the differential law, namely, the law which determines the action of each element of one conductor upon each element of any other conductor or upon itself. He said, let us now resolve this integral action into elementary actions. He did it and he found that each element of the conductor acts upon a pole or upon an element of another conductor with a force which is not exerted in the line connecting the two, but perpendicularly to that line. Other differential laws have been deduced in the last twenty years by Maxwell, Weber and Helmholtz. Some of these laws suppose that there is not only a force perpendicular to the line between the two elements, but also that there is something that tends to turn the element, so that we have to deal here not only with forces, which are determined by laws of mechanics, but with forces that lie entirely outside of that. Therefore we are always to carefully distinguish what we mean by force. Is it purely mechanical force, or is it a force which produces, besides motion, something else? We cannot speak of chemical forces in the same way as we speak of mechanical forces, because mechanical forces are limited by the definition of Newton and they produce nothing but the motion of the mass. Chemical force produces heat; it produces chemical composition and decomposition; it $\mathrm{l}$ roduces light and it may produce motion also. We do not deal here with pure forces such as gravitating force which is always exerted in the line connecting the two points acting upon one another.

The other point which I would like to mention refers to con- 
ductivity. A gentleman said that the rule-I will not call it a law -is that every metal increases its resistance with temperature, and therefore at a certain low temperature it ought not to offer any resistance at all. The fallacy is self-evident. When we speak of a law we do not mean something absolutely true. Clifford has defined its meaning; a law is true only for those conditions under which the particular phenomena which led to the construction of the law were observed. The law of heat, the law of light, the law of electric current were observed; not in a temperature of several million degrees, but in temperatures which we can get ranging from mir us 80 degrees centigrade to perhaps 3000 degrees centigrade, the highest temperature which we could obtain. Therefore the law above those limits may be true or may not be true. Every law may be represented by a certain curve. Where the curve does not run from minus infinity to plus intinity we know a very small space of that curve; the rest we do not know.

Prof. Garver:- - I see I was misunderstood in reference to the conductor. I was speaking of the fact that at first sight it seems that the variation in conductivity due to the temperature in the conductor was at variance with the view that the action took place outside instead of inside the conductor. Since thinking it over I have arrived at what seems to be a plansible answer to my own question, that the conducter, being actuated by heat, the particles are in motion. Now, as the temperature of the conductor goes down, the heat action becomes less and less, and it then approximates to what I believe it is supposed to be-a hole through the medium.

Mr. Nikola Tessa :- I want to suguest a practical idea on which I have not taken a patent, but which is cheerfully offered in case any gentleman wants to patent it. There seems to be no doubt to-day that light and electricity are one and the same, and that between the two there is only a difference in degree. Now, the question resolves itself into how to produce a sufficient number of undulations to equal those of light. We know that if you put two coils in inductive relation and pass through the primary an undulating current you can produce in the secondary two distinct currents. Then from that secondary we can again act in the same manner upon another coil and so on I have figured that at that rate it would require about 23 conversions. If you assume an efficiency of 80 per cent in the tirst you can assume one of 2 per cent. in the last coil which is the twenty-third.

Now there is another thing I wish to mention. That is, that while we are convinced that light and electricity and electromagnetic action are the same, yet there seems to be a difference between light and electricity in that we cannot maintain light in a static state; but we can maintain electricity so. And again light and magnetisn are similar in this respect That when we attempt to store, for instance, magnetism and light-a problem 
which is very interesting and would be very valuable if solvedwe know well that we can magnetize steel and it will hold magnetism indefinitely. We can also magnetize a soft iron bar but it will lose the magnetism soon. Now there seems to be, from what Prof. Anthony has so admirably set forth, a possibility of producing a magnet of such qualities that it would for an indefinite time, say for a year or more, continuously discharge its magnetism after a cessation of the current which energized it. Then we could store electricity. I attempted in my early days to do that, but it does not work. Another thing in regard to light-if we expose certain bodies to an arc light or a very intense source of light we will find that they will take up light for the first moments, but when the light is intercepted it will instantly fall and the little that remains will gradually disappear, and sometimes it will take considerable time, similar to that little remnant of magnetism in the big bar of iron. If we plot the curves in the two cases we would find them similar, which shows that magnetism and light correspond in this way. Whereas we know that electricity can be stored-I understand now the difference which Prof. Anthony has brought out between potential and electro motive force-I mean we can take the condenser or a Leyden jar and store up electricity in any quantity, so to speak, and keep it there for a considerable time, and then discharge it at will. We cannot do so with light nor with magnetism, and yet we know that all these are really one and the same thing with only a difference of degree.

Dr. Pupin:-I would like to ask Prof. Anthony to define the term electromotive force.

Prof. Anthony:- It is one of the most difficult things in the world to give the definition, and I never pretended to any ability at giving definitions. We all know that in a battery there is something that develops an activity. We know that the conductor gets warm. We know that around the conductor there are what we call magnetic forces, and I believe that there is an activity set up in the whole space around it. Now, whatever there is inside of the battery that gets up this agitation I call electromotive force. Furthermore, we know that if we take a wire and move it across the magnetic lines of force there exists something which, if that wire formed a closed circuit, will produce what we call a flow of electricity. We know it is full of electricity, because, under proper conditions the wire will get warm, because also, there are magnetic effects developed by it. There is something takes place-an agitation is set up in that wire, and I consider that something, I do not pretend to define it or to know what it is, but there is something there in consequence of the movement of that wire across these magnetic lines of force that constitutes what we call electromotive force. It is something, to use the language of electrical theory, that puts electricity in motion, and that is the action I referred to in the paper in which 
we may have electricity in motion in a conductor without any difference of potential. Suppose, for instance, I have a perfect circle formed of wire, and then I take a magnet, and suppose I thrust the north pole of the magnet perpendicularly into the very centre of that circle. We know that lines of force cut that circle all around in every part, and when that magnet is thrust through there we know that whatever there is that constitutes a magnetic field acts in some way to set up this current and acts in every part of the wire so that there is a current set up without there being any difference of potential in any place.

DR Pupix:-How would you measure the heat produced in wire? Is that subject to Joule's law ?

Prof. Anthony :-I should say so, certainly.

DR PUPIN:- It is equal to the electromotive force multiplied by the current. What is the electromotive force? It is that which we define mechanically in absolute measures.

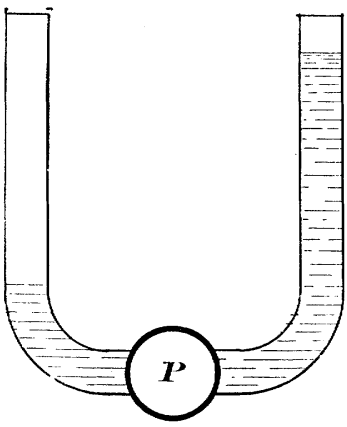

Fig. 3.

Prof. Anthony :- It is whatever sets that current in motion around there.

Dr. Pupin :-Yes, but we know it, we can define it in terms of time, mass and length. It is perfectly definable.

Prof. Anthony :-It is measured in the same terms as difference of potential, of course.

I R. Pupin :- It must be the same.

Prof. Anthony:- I do not think so any more than in difference of level We measure difference of pressure, for instance, by difference of level in the case of water. To illustrate it-here I have $\pi \mathrm{U}$ tube with a pump in it The water is at the same level in both branches. I now set the pump turning and the water falls in this side and goes up in the other. That difference of level will measure the difference of pressure, but that pressure was produced by turning the handle on this pump. Now the turning of the handle I call the water-moving force. This constitutes a difference of potential, a difference of gravity potential which opposes my turning of the handle. (See Fig. 3.) 
DR. PLPIN:-Therefore the work done measures the difference of level.

Prof. Anthony :- It may be measured by the difference of level.

Dr. Puprn:-But the work done measures the difference. The electromotive force is not a force at all in the nsual sense of the word. It is the work done to make the difference of potential and that is what the difference of potential is-the work done.

Prof. Anthony :-Suppose that we do not produce any difference of potential, as in the case of the circle of wire with the magnet thrust into it.

Dr. Pupin :-But we do. That is a well-known problem.

Prof. Axthony :-The difference of potential is equal to the work done. I may just as well say substitute for each element in that wire an intinitely small Daniell element all around. Of course that is practically impossible, but let us suppose so. We can place intinitely small Daniell elements, connecting them together around the circle so that they will form a closed circuit, where is the difference of potential there? If you have them infinitely small so that each Daniell element acts on each element of the wire then I should say there was no difference of potential from one end to the other, and we have the electromotive force of the Daniell element producing the result entirely and nothing else.

Dr. Pupin:-There is a difference of potential between each two poles in every one of those infinitely small cells. So I may say between the ends of each one of the infinitely small elements of the wire there is a difference of potential and these being connected in series the current runs around them.

Prof. Anthony:-Let us take it in another way. Suppose there is a circular tube containing a gas or liquid, whichever you please. Now, can we not conceive of such a thing as something acting simultaneously upon every single element, no matter how many you consider there may be of them, so that every one of those elements will move forward at the same instant, and that there is no difference of pressure anywhere in that case. Now, difference of pressure is what I consider as equivalent to difference of potential. In other words what we call difference of potential electrically is due to a disturbed electrical distribution and until you have a disturbed electrical distribution there is no difference of potential. Now, in this wire where we have a perfect circle and thrust into it the magnetic pole, we act on every element of that wire simultaneously, there is no disturbed condition of potential, and after you have stopped the magnetic pole you can conceive that your electricity has been shifted around through the whole of it without there being a disturbance there-without any difference of potential. You stop the movement of the magnetthe current stops. Your electricity does not fly back again. But let us cut that wire in two and leave an opening in it. Now 
force the magnetic pole through the middle and you tend to make a motion around it in the same way. Electricity is heaped up at one end, making pressure there, and the moment your magnet stops the electricity flies back and produces a current the other way.

Mr. Francis B. Crocker:-It seems to me that Prof. Anthony's last illustration will give us a solution of the problem and reconcile both sides of this controversy. He assumes that the wire circle is opened at a certain point, and if you thrust in a magnet you get at that point the total difference of potential which we could calculate and predict from known electric and magnetic effects Now it seems to me that when the circle is closed, you get that difference of potential spread over the entire con ductor which produces the current through that resistance. It is heaped up at the point of opening because it cannot pass that point, there being no conductor there. But it appears to me that, if we close the circle at that point and make it uniform as regards resistance, the potential is distributed uniformly around the entire circle. But even when distributed in that way it produces the same effect as it would if it were concentrated, and it would seem that although there is no difference of potential which can be detected ye the same total difference of potential really exists throughout the circle. In other words the integral of the difference of potential is the same in both cases, because the current in the circuit and the Joule effect and all those other electrical phenomena will be precisely those due to the difference of potential which we get when we open the circuit. Theoretically the way to treat such a case is to differentiate-take a certain element and treat it by itself. Taking a certain element there, we have a certain difference of potential, a certain resistance and a certain resulting current. Take for instance that circle and assume it for the purpose of the hypothesis to be divided into a thousand equal portions. Then we have one 1000th of the total difference of potential and one 1000th of the resistance in each element, and therefore we have the same current as with a thousand times the electromotive force and a thousand times the resistance. 'This seems to me to be merely a particular case-an electrical paradox.

Mr. Wolcotr :-Mr. Crocker's idea of the circulation of current there is a good deal like the relation of east and west on the globe. We can have a point west of New York and by going in the other direction it will be east of New York. If we take Mr. Crocker's view and integrate the difference of potential all around the ring, when we get back to the starting point, that point has two potentials, and so it must be It is like any other cyclic region. One of the most familiar cases of that is the magnetic. potential. In an infinitely straight conductor the potential at any point has an infinite number of values, but it is only on some such conception as that, that you can consider there is any difference of potential. Each point has a potential different from itself. 
That is by going around until you come back to the same point you have increased the potential, by the cyclic constants of the curve.

Mr. Kennelly :-I think we are very much indebted to Prof. Anthony for having called attention to one of the leading misconceptions of the day. There is a constant conflict between difference of potential and electromotive force, and I think nothing can be better in a society of this kind than to settle such confusions. I think Prof Anthony has given us a very clear solution in this way, that whatever produces a current is electromotive force; that electromotive force may or may not be accompanied by a difference of potential. We do not recognize potential as we recognize current. We are led to it by a series of abstractions. Potential was introduced on a purely theoretical and mathematical basis. The potential function first existed in gravitation. It was introduced into electricity by similarities which exist between gravitation laws and electrical laws in their mathe matical form, and the potential is something which causes work to be done, in virtue of which work can be done. The potential is measured by the work done in bringing a unit of electricity to the point whose potential is to be determined.

Dr. PuPIN :- I do not agree with the last speaker, that potential is a mathematical conception any more than I would agree with him that work is a mathematical conception. Who would say that to raise a barrel of flour to the gallery above us here is a mathematical conception? To charge a conductor with a certain amount of electricity, we must do a certain amount of work. If we do not stick to this term work, we cannot make a step of progress, because we cannot use the principle of the conservation of energy. Potential is not a mathematical conception. Potential is as concrete as the fact that I am standing here. It is the amount of work done. He said that difference of potential is something which causes work to be done, and a little while before that, he said that electromotive force is the cause of the electric current. Now these two things seem to me to be one and the same, because work must be done to cause the current to flow ; but I will not discuss the subject any more, seting that my colleague has expounded my idea of this circular conductor in a very nice and masterly way.

The Chairman :- We certainly have had one of the most interesting evenings in the history of the Institute. I think the discussion has been very suggestive in many ways.

On the 18th of February, will occur the next meeting of the Institute, when a paper will be read by Mr. William Maver, Jr. and the topic will be, "The Practical Working of New York's Electrical Sub-ways." You may expect that to be an extremely interesting paper, full of surprises.

Adjourned. 